c.643C > T (p.R215X), splicing c.1539+1G >A and frameshift duplication c.453_456dup (p.S153Vfs*5). The last one was not previously described in the HGMD database.

Clinical and genetic features were described in Russian patients with dystroglycanopathy due to POMGNT1 mutations.

\section{CDHR1 AND RGR MUTATIONS IN TWO PATIENTS WITH RETINITIS PIGMENTOSA}

A Bobinec*, A Meašić, I Sansović, M Kero, L Boban, I Barišić. Department of Medical Genetics and Reproductive Health, Children's Hospital Zagreb, Scientific Centre of Excellence for Reproductive and Regenerative Medicine (CERRM), University of Zagreb School of Medicine, Zagreb, Croatia

\subsection{6/archdischild-2021-europaediatrics.95}

Retinitis pigmentosa (RP) is a group of inherited retinal disorders characterized by progressive photoreceptors and pigment epithelial cells dysfunction. Patients initially present with nyctalopia from rod photoreceptor loss, progress to tunnel vision and ultimately experience central vision loss. In majority of cases (70-80\%) RP is nonsyndromic and may be inherited in an autosomal dominant, autosomal recessive and X-linked recessive manner. The most frequent forms of syndromic RP are Usher and Bardet-Biedl syndrome. It is estimated that RP affects 1 in 3.000 to 1 in 5.000 people worldwide and so far mutations in more than 300 genes have been associated with development of this disorder.

We present two patients with referral diagnosis of RP in whom we performed molecular analysis of clinically relevant genes using NGS. In both patients we detected mutations in CDHR1 and RGR genes. First patient is a 36-year old female homozygous for the p.Ile841Serfs119 mutation in CDHR1. Variations in this gene can cause autosomal recessive retinopathy with clinical manifestations ranging from RP to cone-rod dystrophies. This patient is also homozygous for p.Ser66Arg mutation in RGR gene that can cause autosomal recessive/ dominant RP type 14. Second patient is a 39-year old male with two heterozygous mutations: p.Ile841Serfs119 and p. His206Thrfs61 in CDHR1 gene.

This patient is also heterozygous for p.Ser66Arg mutation in RGR gene. Arno et al. have previously described 6 unrelated families in whom homozygous mutations p.Ile841Serfs119 in CDHR1 and p.Ser66Arg in RGR genes were detected. Four of these families were of Albanian origin as is our female patient.

Our findings confirm the hypothesis of Arno et al. that homozygous and compound heterozygous mutations in the CDHR1 gene may co-segregate with mutations in the RGR gene that are likely to have a modifying effect on the phenotype. This constellation is common in the Albanian population indicating a founder effect.

This work was supported by Scientific Center of Excellence for Reproductive and Regenerative Medicine and by the European Union through the European Regional Development Fund, under grant agreement No. KK.01.1.1.01.0008, project „Reproductive and Regenerative Medicine - Exploring New Platforms and Potentials.

\section{6 HOMOZYGOUS ABCG8 MUTATION IN A 14-YEAR-OLD BOY WITH SITOSTEROLEMIA}

Ana-Maria Meašić ${ }^{*}$ Adriana Bobinec, Ivona Sansović, Ljubica Boban, Ana Močić Pavić, Silvija Pušeljić, Ingeborg Barišić. Department of Medical Genetics and Reproductive Health, Children's Hospital Zagreb, Scientific Centre of Excellence for Reproductive and Regenerative Medicine (CERRM), University of Zagreb School of Medicine, Zagreb, Croatia

\subsection{6/archdischild-2021-europaediatrics.96}

Introduction Sitosterolemia is a rare, inherited, autosomal recessive lipid disorder characterized by the accumulation of plant sterols and cholesterol in plasma and tissues as a consequence of hyperabsorption and impaired biliary secretion of sitosterol. It is caused by mutations in ABCG5 and/or ABCG8 genes that encode a pair of $\mathrm{ABC}$ half transporters and form a heterodimer (ABCG5/G8), which then traffics to the surface of hepatocytes and enterocytes and promotes the secretion of sitosterol into the bile and the intestinal lumen. Here we report on a 14-year-old boy with hypercholesterolemia, failure to thrive and short stature.

Methods Patient was referred to use due to suspicion of familial hypercholesterolemia (FH). His father has hypercholesterolemia and arterial hypertension, grandfather on father's side also has arterial hypertension and his mother and grandmother on mother's side have thrombocytopenia. One of his two younger brothers has unilateral perceptual hearing loss, hypercholesterolemia, thrombocytopenia and short stature. NGS analysis of clinical exome (CES, Clinical Exome Sequencing) was performed using peripheral blood DNA obtained from patient and his parents. CES analysis in affected brother is ongoing.

Results CES revealed homozygous missense mutation ABCG8: c.584T >A, p.(Leu195Gln) in patient. His parents are heterozygous carriers of the same mutation. According to ACMG/ AMP classification and publications data this variant is classified as likely pathogenic.

Discussion and Conclusion Leucine at codon 195 is highly conserved among all five human ABCG transporters. This mutation is localized within the nucleotide-binding domain (NBD) that powers the transport of substrates by binding and hydrolyzing ATP molecules. Phenotypically, sitosterolemia is very heterogeneous. It includes hypercholesterolemia, accelerated atherosclerosis, premature coronary artery disease, xanthomas, hemolytic anemia, stomatocytes, macrothrombocytopenia, arthraligia, splenomegaly and elevated liver function test. Because of this it is considered extremely underdiagnosed with over 100 cases reported so far. According to rough estimations the frequency could be at least 1 in 200.000 individuals. It is also considered a recessive disorder, but recent studies have revealed that heterozygous carriers also exhibit milder manifestations as seen in this patient's parents. Sitosterolemia is also often misdiagnosed as FH and mistreated with statins that are partially or completely ineffective. In conclusion, sitosterolemia should be considered in children with hyperlipidemia, especially with a significant hypercholesterolemia and increased low-density lipoprotein levels.

This study was supported by CERRM, Republic of Croatia, and by the EU through ERDF, under grant agreement No. KK.01.1.1.01.0008, project Reproductive and Regenerative Medicine - Exploring New Platforms and Potentials'. 